\title{
Confidence in assessing the effectiveness of bath treatments for the control of sea lice on Norwegian salmon farms
}

\author{
Daniel F. Jimenez ${ }^{\mathrm{a}, *}$, Peter A. Heuch ${ }^{\mathrm{a}}$, Crawford W. Revie ${ }^{\mathrm{b}}$, George Gettinby ${ }^{\mathrm{c}}$ \\ a Norwegian Veterinary Institute, P.O. Box 50 Sentrum, 0106 Oslo, Norway \\ ${ }^{\mathrm{b}}$ Atlantic Veterinary College, University of Prince Edward Island, Charlottetown, Canada \\ c University of Strathclyde, Glasgow, Scotland, UK
}

\section{A R T I C L E I N F O}

\section{Article history:}

Received 2 March 2012

Accepted 22 March 2012

Available online 30 March 2012

\section{Keywords:}

Atlantic salmon

Bath treatments

Efficacy

Sea lice

Lepeophtheirus salmonis

\begin{abstract}
A B S T R A C T
The salmon louse Lepeophtheirus salmonis is the most important ectoparasite of farmed salmonids in the Northern hemisphere, having a major economic and ecological impact on the sustainability of this sector of the aquaculture industry. To a large extent, control of $L$. salmonis relies on the use of topical delousing chemical treatments in the form of baths. Improvements in methods for the administration and assessment of bath treatments have not kept pace with the rapid modernization and intensification of the salmon industry. Bath treatments present technical and biological challenges, including best practice methods for the estimation of the effect of lice treatment interventions. In this communication, we compare and contrast methods to calculate and interpret treatment effectiveness at pen and site level. The methods are illustrated for the calculation of the percentage reduction in mean abundance of mobile lice with a measure of confidence. Six different methods for the calculation of confidence intervals across different probability levels were compared. We found the quasi-Poisson method with a $90 \%$ confidence interval to be informative and robust for the measurement of bath treatment performance.
\end{abstract}

(C) 2012 Elsevier B.V. All rights reserved.

\section{Introduction}

The salmon louse Lepeophtheirus salmonis constitutes a persistent parasite infection and a constant source of production losses in the culture of salmonids in the Northern hemisphere. Control of the parasite relies on the use of chemical treatments administered topically in bath treatments, and orally in the feed. In recent years, the use of bath treatments has increased due to the apparent development and spread of resistance to the widely used in-feed treatment, emamectin benzoate (Jones et al., in review; Lees et al., 2008; NFSA, 2010a). There is currently no standardized way to estimate and report treatment effectiveness in net pen fish farms, unlike the evaluation of efficacy of parasiticidal treatments in terrestrial species (Coles et al., 1992). In this paper, we propose a method and guidelines for evaluation and interpretation of the effectiveness of bath treatments. The method involves calculation of a point estimate of the percentage reduction in the number of mobile lice, with the associated uncertainty expressed as a confidence interval.

It is crucial to assess the true effect of bath treatments and thus provide a basis for detection and correction of intrinsic and external factors that limit the use of these treatments in the field.

\footnotetext{
* Corresponding author. Tel.:+47 232164 80; fax:+47 23216485 . E-mail address: Daniel.jimenez@vetinst.no (D.F. Jimenez).
}

However, current legislation neither provides grounds for the selected $90 \%$ margin, nor guidelines for the evaluation of treatment effect (NFSA, 2010b; NFSA, 2011). The proposed method is a more realistic approach to evaluation and interpretation of the true effect of treatment. It will be more sensitive in identifying those pens and sites which are below the recommended level of efficacy and decrease the associated risk of treatment resistance developing (Denholm et al., 2002).

\section{Materials and methods}

\subsection{Study population}

The dataset originates from a study conducted (Heuch et al., 2003) on salmon and rainbow trout farming sites located on the Norwegian coast. Sites with data on samplings performed the week prior to and after delousing treatment were selected. In this subset, a total of 1002 fish from five different sites and 18 pens were inspected on 24 treatment occasions between September 1997 and November 1999. For treatment evaluation, we extracted counts of mobiles within the week prior and after treatment. The majority of pens (21/24) had a single treatment and the three remaining pens in the same site were treated in three different occasions. The dataset included counts for chalimus, pre-adult, adult male and adult females of $L$. salmonis of 20 or more individual fish from each pen on each sampling occasion. Counts were performed at weekly, biweekly or monthly intervals 
depending on site and study period. Bath treatments were undertaken with deltamethrin (AlphaMax®, Alpharma) using skirts. At the time, this was the most popular and effective delousing treatment available (Denholm et al., 2002).

We describe the distribution of mobile lice (pre-adult and all adult sea lice stages) both at pen and site level in the week prior to and 1 week after treatment. The distribution of mobiles tends to vary depending on mean abundance of $L$. salmonis (Heuch et al., 2011). For Poisson regression, goodness of fit of the model was tested using Pearson's chi-square. Empirical data were fit to the Poisson, quasiPoisson and negative binomial distributions with maximum likelihood using the 'fitdist' and the 'vcd' packages available with $\mathrm{R}$ software (Friendly, 2000; Venables and Ripley, 2002; Vose, 2008). Overdispersion is characteristic of most parasitic infections (Anderson and May, 1992) including L. salmonis (Baillie et al., 2009; Heuch et al., 2011). Here, overdispersion refers to the variance of mobiles being larger than the mean. An indication of overdispersion is when the deviance measure of the goodness-of-fit for full model (standardized residuals) exceeds its degrees of freedom (Venables and Ripley, 2002).

\subsection{Calculation of estimates and confidence interval for the percent reduction in count of mobiles}

The percentage treatment effectiveness is calculated as $100 *\left(\frac{\mu_{0}-\mu_{1}}{\mu_{0}}\right)$, where $\mu_{0}$ and $\mu_{1}$ are the mean abundances of $L$. salmonis before and after treatment, respectively.

For calculation of treatment effectiveness, we have used mobiles (pre-adult and adult stages). Counts of chalimus stages were not included because this stage is known to be relatively insensitive to most chemical treatments used in baths (Burka et al., 1997). Due to their small size $(0.7-2.7 \mathrm{~mm})$ they are also more difficult to see when examining the fish. Hence, counts of chalimus are often underestimated on the farm (Beamish et al., 2005; Schram, 1993).

Calculation of treatment effectiveness was made both at individual pen and site level. At site level, we aggregated counts of mobiles for all treated pens to obtain a single estimate of treatment effectiveness. Confidence intervals for the ratio of two means were calculated using approximate methods (Chaudhary and Stearns, 1996; Cimermanová, 2007). Six methods were used to calculate confidence intervals with the 'pairwiseCl' package available in $\mathrm{R}$ ( $R$ Development Core Team, 2008). These included two methods for continuous data: a two-sample $t$-test with Fieller confidence intervals assuming heteroscedasticity (Hasler and Hothorn, 2008; Hasler et al., 2008), and the ratio of two means assuming a lognormal distribution (log-normal ratio) (Chen and Zhou, 2006). Three methods for count data were also evaluated, the Poisson, quasi-Poisson and negative binomial methods (Venables and Ripley, 2002; Zeileis et al., 2008). The last method is a percentile bootstrap confidence interval using the Harrell-Davis method (Harrell and Davis, 1982). The original dataset was modified for calculation of the confidence intervals for the twosample $t$-test and the log-normal ratio. For the latter, 0.1 was added to all data; in the former a value of 0.1 was added to one fish if none of the fish in the sample were infected with mobiles (Tukey, 1977).

A two sided confidence interval was chosen to state the precision of the estimate of treatment effectiveness (Steiger and Fouladi, 1997). We examined confidence levels of $70 \%, 80 \%, 90 \%$ and $95 \%$ to study their effects on the interval widths. The confidence level is dictated by criteria which include confidence and informativeness or decisiveness (Senn, 2007; Smithson, 2003).

\subsection{Treatment vs control abundance for effectiveness}

The effects of synthetic pyrethroids have been previously investigated. Several studies have shown that adult and pre-adult stages of $L$. salmonis are highly susceptible to synthetic pyrethoids (Hart et al., 1997).

The required minimum margin is decided by the user, although common practice is often used such as in trials of human antibiotics, where a $10 \%$ or $20 \%$ difference compared to the efficacy of the reference treatment is used (FDA, 2010). The same margin is recommended for the approval of new antiparasitic treatments in terrestrial animals (Burridge et al., 2010; CVMP, 2001; Schall and Luus, 2011). The Norwegian Food Safety Authority (NFSA), states that a $90 \%$ reduction is satisfactory (NFSA, 2010b; NFSA, 2011).

Treatment effectiveness is here measured relative to a chosen standard of treatment efficacy which could be any value. In the case of synthetic pyrethroids, prior studies have shown the average percent reduction in pre-adults and adult females was in the 95 to $99 \%$ range (Hart et al., 1997). In this case, we have followed the guidelines laid out by the NSFA. We classify the treatment effect as acceptable or unacceptable (failure). Treatments were considered acceptable when the calculated lower confidence limit is equal to or higher than $90 \%$. When the calculated lower limit is below the $90 \%$ minimum margin of efficacy, the treatments were considered unacceptable.

\section{Results}

\subsection{Descriptive statistics}

In the extracted subset of data, average counts of mobiles per fish before treatment were 4.6 (0.1 to 16.5) at pen and 4.9 (0.3 to 10.1) at site level (Table 1 ). The calculated mean and variance were similar at mobile counts around $10-12$ per fish (Fig. 1a). In $60 \%(6 / 10)$ of the sites and $75 \%(18 / 24)$ of the pens, the Poisson distribution fits the

Table 1

Mean and variance of the counts of $L$. salmonis mobiles (pre-adult and adult stages) before $(\mathrm{t} 0)$ and after treatment ( $\mathrm{t} 1$ ) according to site, treatment date (month, year) and pen.

\begin{tabular}{|c|c|c|c|c|c|c|}
\hline Site & $\begin{array}{l}\text { Treatment } \\
\text { date }\end{array}$ & $\begin{array}{l}\text { Pen } \\
\text { number }\end{array}$ & $\begin{array}{l}\text { Mean } \\
(\mathrm{t} 0)\end{array}$ & Variance(t0) & $\begin{array}{l}\text { Mean } \\
(\mathrm{t} 1)\end{array}$ & $\begin{array}{l}\text { Variance } \\
\text { (t1) }\end{array}$ \\
\hline 1 & 7/1997 & 30 & 10.1 & 16.2 & 0 & 0 \\
\hline 1 & 7/1997 & Site average & 10.1 & 16.2 & 0 & 0 \\
\hline 1 & 8/1998 & 22 & 11.7 & 18.3 & 3.9 & 9.5 \\
\hline 1 & $8 / 1998$ & 27 & 5.3 & 13.5 & 1.9 & 2.8 \\
\hline 1 & 8/1998 & Site average & 8.7 & 26.2 & 2.9 & 7 \\
\hline 2 & 8/1997 & 1 & 16.5 & 297 & 0.1 & 0.1 \\
\hline 2 & 8/1997 & 2 & 6.4 & 8.3 & 0.9 & 1.7 \\
\hline 2 & 8/1997 & 3 & 12.6 & 75.5 & 0.6 & 0.9 \\
\hline 2 & $8 / 1997$ & Site average & 11.8 & 140.4 & 0.5 & 1 \\
\hline 3 & $10 / 1998$ & 1 & 0.4 & 0.2 & 0 & 0 \\
\hline 3 & $10 / 1998$ & 6 & 0.1 & 0.2 & 0 & 0 \\
\hline 3 & $10 / 1998$ & 7 & 0.5 & 0.5 & 0 & 0 \\
\hline 3 & $10 / 1998$ & Site average & 0.3 & 0.4 & 0 & 0 \\
\hline 3 & 7/1999 & 1 & 4.3 & 10.7 & 0.3 & 0.4 \\
\hline 3 & $7 / 1999$ & 6 & 3.6 & 3.2 & 0.1 & 0.1 \\
\hline 3 & 7/1999 & 7 & 3.7 & 6.1 & 0.2 & 0.1 \\
\hline 3 & 7/1999 & Site average & 3.9 & 6.6 & 0.2 & 0.2 \\
\hline 3 & 8/1999 & 1 & 1.8 & 2.3 & 0.1 & 0.1 \\
\hline 3 & 8/1999 & 6 & 1.5 & 2.1 & 0.3 & 0.2 \\
\hline 3 & $8 / 1999$ & 7 & 4.4 & 5.7 & 0.3 & 0.2 \\
\hline 3 & 8/1999 & Site average & 2.5 & 5 & 0.2 & 0.2 \\
\hline 4 & 8/1998 & 5 & 1 & 1.3 & 0 & 0 \\
\hline 4 & $8 / 1998$ & Site average & 1 & 1.3 & 0 & 0 \\
\hline 5 & 3/1999 & 7 & 0.9 & 0.9 & 0 & 0 \\
\hline 5 & $3 / 1999$ & 9 & 0.7 & 1.1 & 0 & 0 \\
\hline 5 & 3/1999 & 12 & 0.8 & 0.6 & 0 & 0 \\
\hline 5 & 3/1999 & Site average & 0.8 & 0.8 & 0 & 0 \\
\hline 5 & $7 / 1999$ & 21 & 4.6 & 5.4 & 0.9 & 3.3 \\
\hline 5 & 7/1999 & 24 & 6.8 & 5 & 0.1 & 0.1 \\
\hline 5 & 7/1999 & 26 & 4.7 & 3.4 & 0 & 0 \\
\hline 5 & $7 / 1999$ & Site average & 5.3 & 5.5 & 0.3 & 1.2 \\
\hline 5 & 8/1999 & 20 & 4.2 & 2.3 & 0 & 0 \\
\hline 5 & 8/1999 & 25 & 4.2 & 3.3 & 0.4 & 0.5 \\
\hline 5 & 8/1999 & Site average & 4.2 & 2.7 & 0.2 & 0.3 \\
\hline
\end{tabular}


A

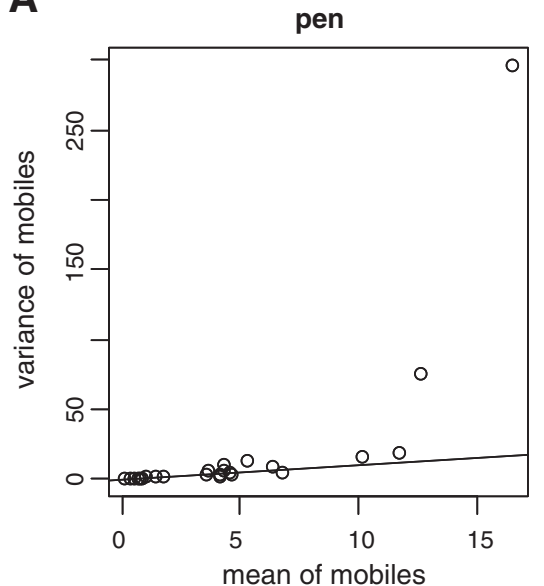

B

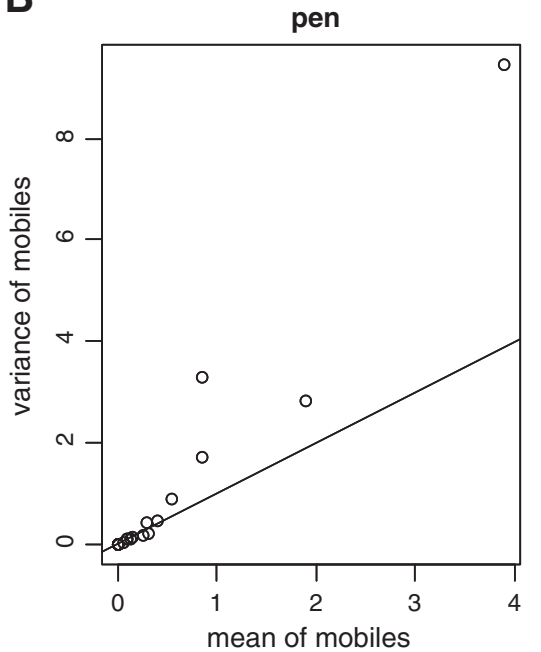

site

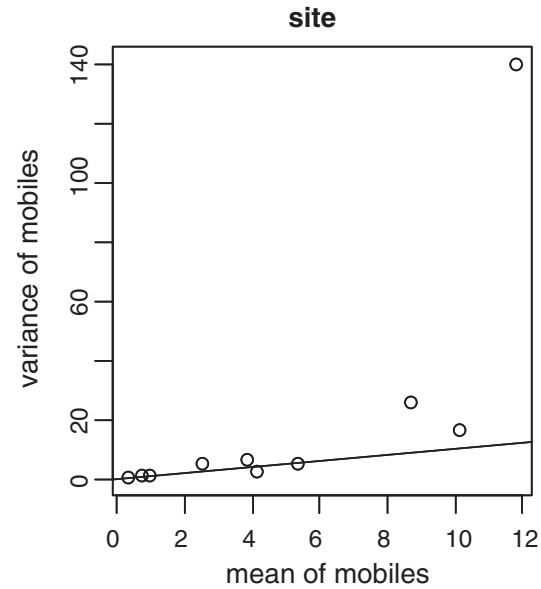

site

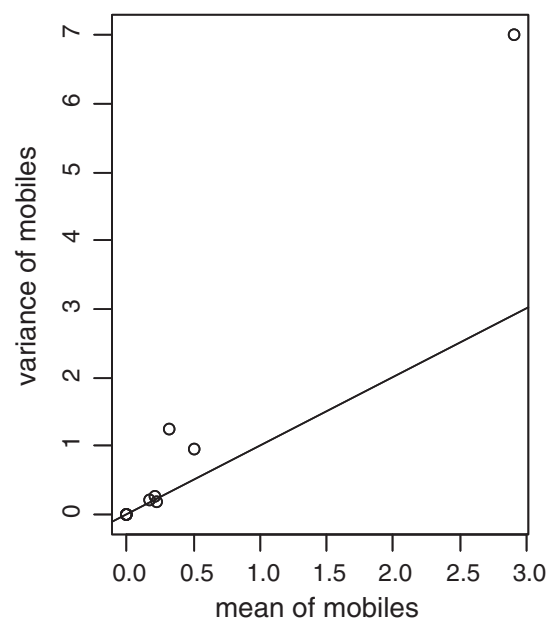

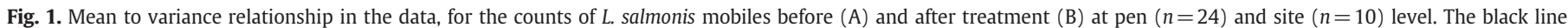

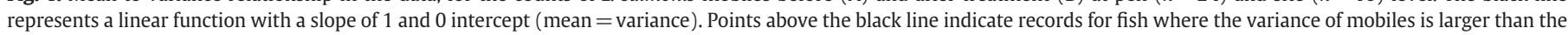
mean (overdispersion).

empirical data well (chi-square goodness of fit test for a Poisson distribution, $p>0.05$ ). After treatment (Fig. 1b), counts of mobiles are dominated by zero values and neither the Poisson nor negative binomial fits the empirical data well due to the excess of zeros present.

\subsection{Calculation of confidence intervals using different methods width $\mathrm{CI}$}

Six methods were used to calculate the 95\% confidence intervals for the estimates of treatment effectiveness at pen (Fig. 2) and site levels (Fig. 3): the t-test, the log-normal ratio, the Harrell-Davis (bootstrap), the Poisson, the quasi-Poisson and the negative binomial. The widths of the confidence intervals varied depending on the method used. The Harrell-Davis method produced the narrowest interval (Figs. 2 and 3). The widest intervals were obtained with the log-normal method. The widths of the confidence interval were similar for the Poisson, the $t$-test, the quasi-Poisson and the negative binomial. For any of these methods the widths of the confidence interval fell between those of the log-normal ratio and the bootstrap method. The point estimates of treatment effectiveness calculated with the $t$-test, log-normal and Harrell-Davis methods were slightly different (Figs. 2 and 3 ) as a result of modification of the original dataset ( $t$-test and log-normal method) or the method for calculation (Harrell-Davis).

Calculations using the log-normal method produced a large number of treatment failures, indeed $29.1 \%$ (7/27) of the pen-level treatments were acceptable. In contrast, bootstrapping results gave the largest number of acceptable treatments (75\%) and the least number of failures (25\%) (Tables 2 and 3).

Methods for count data (Poisson, quasi-Poisson and negative binomial) produced comparable results. At pen level, the quasiPoisson produced less failed treatments compared to the Poisson and negative binomial (Tables 2 and 3). At site level 70\% (7/10) to 80\% (8/ 10) of treatments were acceptable. One more site failed the treatment with the quasi-Poisson compared to the Poisson and negative binomial. The three sites where treatment failed had a large proportion of treatment failures at pen level ranging from $67 \%(2 / 3)$ to $100 \%(2 / 2$ and $3 / 3)$ (data not shown).

We compared the width of the confidence intervals calculated with the quasi-Poisson method at four different confidence levels: 70\%, 80\%, 90\% and 95\%. Obviously smaller confidence levels reduced the width of the confidence interval (Figs. 4 and 5) and therefore tended to reduce the number of failed treatments (Tables 4 and 5).

Evaluation of treatments was carried out using the quasi-Poisson method with a $90 \%$ confidence level. At individual pen level, $21 \%$ of the pens had an estimate of effectiveness below 90\%, and this increased two-fold (46\%) when the evaluation was made using confidence intervals. A threefold increase (from 10\% to 30\%) in the number of failed treatments was shown at site-level when using confidence limits compared to the point estimate of treatment effectiveness. 

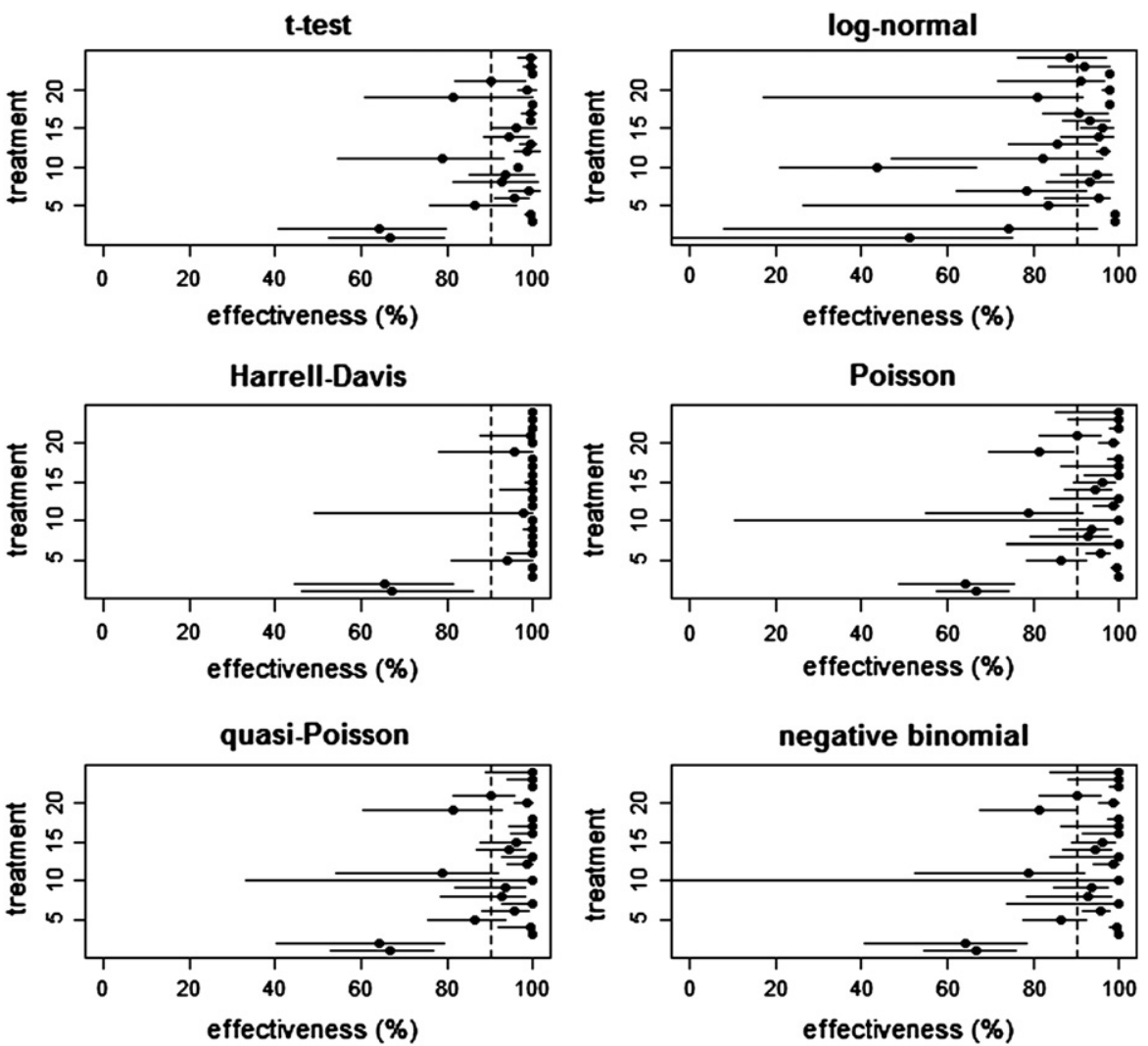

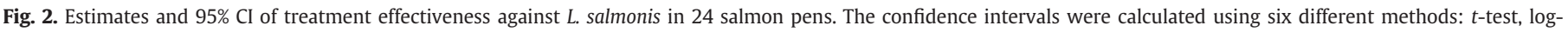
normal, Harrell-Davis, Poisson, quasi-Poisson and negative binomial.

t-test

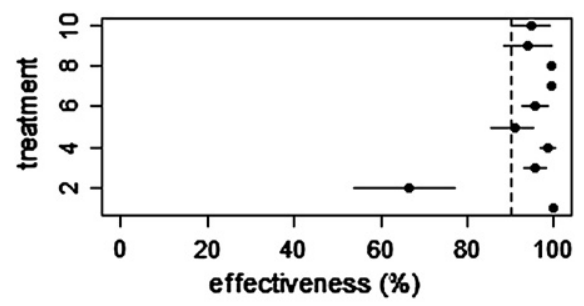

Harrell-Davis

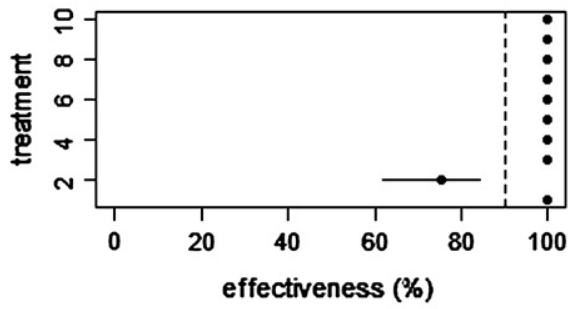

quasi-Poisson

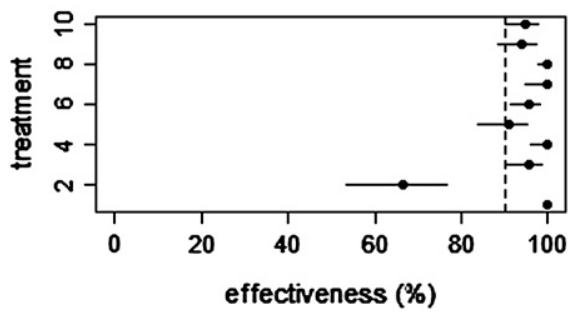

log-normal

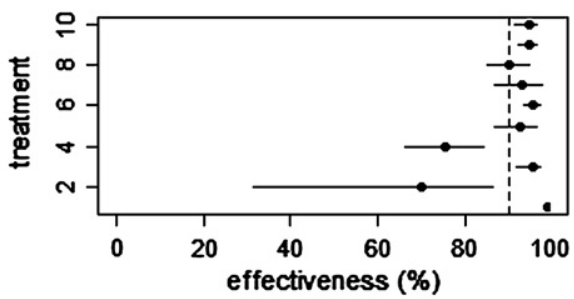

Poisson

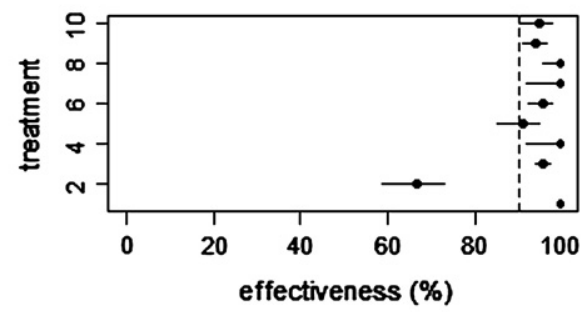

negative binomial

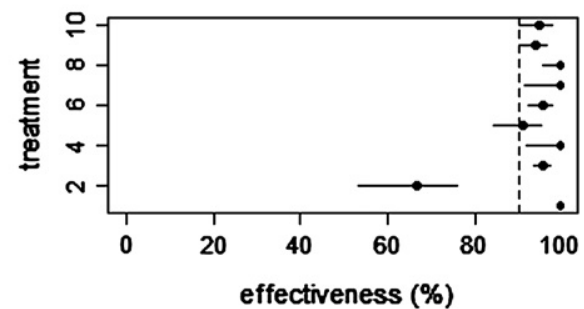

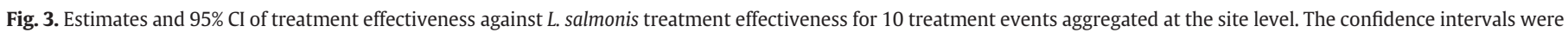
calculated using six different methods: $t$-test, log-normal, Harrell-Davis, Poisson, quasi-Poisson and negative binomial. 
Table 2

Number of treatments against $L$. salmonis at pen level $(n=24)$ that were acceptable (A) at $90 \%$ effectiveness and unacceptable ( $U$ ) according to the methods described in the text. Estimates and $95 \% \mathrm{CI}$ were calculated with six methods.

\begin{tabular}{lllllll}
\hline \multirow{2}{*}{ Result } & \multicolumn{6}{l}{ Method for calculation of CI } \\
\cline { 2 - 7 } & -test $^{\mathrm{a}}$ & log-normal & Harrell-Davis & Poisson & Quasi-Poisson & $\begin{array}{l}\text { Negative } \\
\text { binomial }\end{array}$ \\
\hline $\mathrm{A}$ & 14 & 7 & 19 & 8 & 11 & 8 \\
$\mathrm{U}$ & 9 & 17 & 5 & 16 & 13 & 16 \\
\hline
\end{tabular}

a The two-sample $t$-test could not compute a 95\% confidence interval for treatment performed at Site 3 (Pen 6) on 10/1998.

\section{Discussion}

The continued use of parasitic treatments has resulted in the development and spread of resistance for common drugs such as organophosphates (Fallang et al., 2004; Jones et al., 1992; Tully and McFadden, 2000), avermectins (Jones et al., in review; Lees et al., 2008); pyrethroids (Sevatdal and Horsberg, 2003) and hydrogen peroxide (Treasurer et al., 2000). The growing awareness of drug resistance emergence in certain sea lice populations and the need for information on their occurrence and spread have increased the requirements for standardized methods of treatment evaluation.

Currently, evaluation of treatment is carried out by comparing the point estimate of treatment effectiveness without confidence limits to a pre-specified fixed value. Evaluation of treatment efficacy in this manner has limitations such as uncertainty in the estimate of treatment efficacy. Hence, it is desirable to calculate the variability in efficacy as estimated, through the use of confidence intervals. A potential problem for the calculation of CIs is the lack of control or non-treated groups of fish, as this precludes a direct method for calculation of confidence intervals (Coles et al., 1992). Several solutions were proposed by Fieller and others, who developed theorems to calculate a confidence interval from a ratio of two means (Chaudhary and Stearns, 1996; Cimermanová, 2007).

We have calculated and compared six methods for calculation of generalized confidence intervals. The log-normal ratio produced the widest confidence interval and bootstrapping the narrowest intervals. These two and the $t$-test produce an estimate of treatment effectiveness that is slightly different from that calculated using the formula in Section 2.2, and therefore were considered inferior to the ones used for count data. The Poisson, quasi-Poisson and negative binomial are preferred because they are more appropriate for count data. All three methods give confidence intervals with similar characteristics.

Using CIs, we here propose basic guidelines for calculation and evaluation of topical treatments against L. salmonis, so that data on the effectiveness of treatments as well as on the epidemiology of drug resistance in sea lice can be more accurately determined.

In our data, the distributions of mobiles prior to treatment fit the Poisson distribution when the average number of mobiles is below ten mobiles per fish. This is in agreement with published studies in Norwegian locations (Heuch et al., 2011). Salmon lice can exhibit overdispersion (Heuch et al., 2011). However, overdispersion is likely to occur at site level due to large differences between levels of mobiles among pens (Heuch et al., 2011; Revie et al., 2007).

Overdispersion can cause underestimation of the standard error and confidence intervals which are too narrow, resulting in wrong inference for the treatment effect. Both the negative binomial and quasi-Poisson are commonly used for data with unbounded positive range whose sample variance is larger than the mean (Ver Hoef and Boveng, 2007). For this reason, we have selected the quasi-Poisson method. The quasi-Poisson produces parameter estimates equivalent to the Poisson, where the mean is equivalent to that obtained using
Table 3

Number of treatments against $L$. salmonis at site level $(n=10)$ that were acceptable (A) at $90 \%$ effectiveness and unacceptable ( $U$ ) according to the methods described in the text. Estimates and 95\% $\mathrm{CI}$ of treatment effectiveness were calculated with six methods.

\begin{tabular}{lllllll}
\hline \multirow{2}{*}{ Result } & \multicolumn{6}{l}{ Method for calculation of $\mathrm{CI}$} \\
\cline { 2 - 7 } & t-test & log-normal & Harrell-Davis & Poisson & Quasi-Poisson & $\begin{array}{l}\text { Negative } \\
\text { binomial }\end{array}$ \\
\hline $\mathrm{A}$ & 7 & 5 & 9 & 8 & 7 & 8 \\
$\mathrm{U}$ & 3 & 5 & 1 & 2 & 3 & 2 \\
\hline
\end{tabular}

the Poisson and the variance is calculated as a multiplicative constant of the mean. This results in standard errors that are larger than those of the Poisson. Quasi-likelihood methods are based on generalized linear model principles and allow calculation of parameter estimates based on a specification of the mean and variance of the model observations without specification of an underlying log-like function (Hilbe, 2011).

Treatment effectiveness was calculated and compared at pen and site level with four different confidence levels. The proportion of acceptable treatments is larger at site compared to pen at the same confidence level. At pen level, disagreement was seen in four out of 24 treatments between the $70 \%$ and $95 \%$ confidence levels. In three out of four of these pens, treatments were unacceptable at the $95 \%$, but acceptable at the $80 \%$, and belonged to sites where treatment effect on the site and adjacent pens were also acceptable (at the 95\% confidence level). In the fourth pen, treatment was acceptable only at the $70 \%$ level, but failed at higher confidence levels both on the site and pen. The best agreement between the treatment effect at site and pen level was achieved at the $90-95 \%$ and $80 \%$ confidence level, respectively. These results are not surprising considering that more data were available, and therefore, less uncertainty and higher precision were found on site versus pen treatments. In this case, it could be justified to use a high confidence level but the choice of confidence level should be dictated by the relative incentives for accuracy and precision in each field situation. Yaniv and Foster (1997) have suggested that the precision of uncertain judgments involves a trade-off between these two competing objectives. Selection of a particular method and confidence level should be further investigated due to the limited availability of data and the substantial differences in today's production management in aquaculture.

Treatment results are classified in two categories, acceptable and unacceptable. We have used a fixed margin for evaluation of treatments since the use of a placebo or a non-treatment group is not possible in field treatments. The expected treatment outcome was equal to or higher than a $90 \%$ reduction in mean abundance of mobiles after treatment, based on prior studies (Hart et al., 1997). A non-inferior margin was initially set at $90 \%$ as recommended in both the guidelines on the efficacy requirements for new parasiticides (CVMP, 2004; Marchiondo et al., 2007) and regulations set by the Norwegian Food Safety Authority (NFSA). The NFSA defines as failures those delousing treatments with effectiveness below $90 \%$ (NFSA, 2011) which must be reported, and further investigated to evaluate possible drug resistance (Directorate of Fisheries, 2009; NFSA, 2010b).

A larger number of treatments would have been deemed to be "failures" if the confidence interval approach had been used rather than a point estimate of treatment effectiveness. Here the lower confidence limits were below the $90 \%$ effectiveness level in the majority of pen treatments. This result is not surprising considering that treatments performed with skirts are much less effective than with full tarpaulin (Corner et al., 2011; Fridell, 2009; SEARCH Consortium, 2006). It is also possible that treatments were limited to a group of pens instead of all pens on the site, which will negatively impact the effect of treatment both at pen and site level. Regardless, 

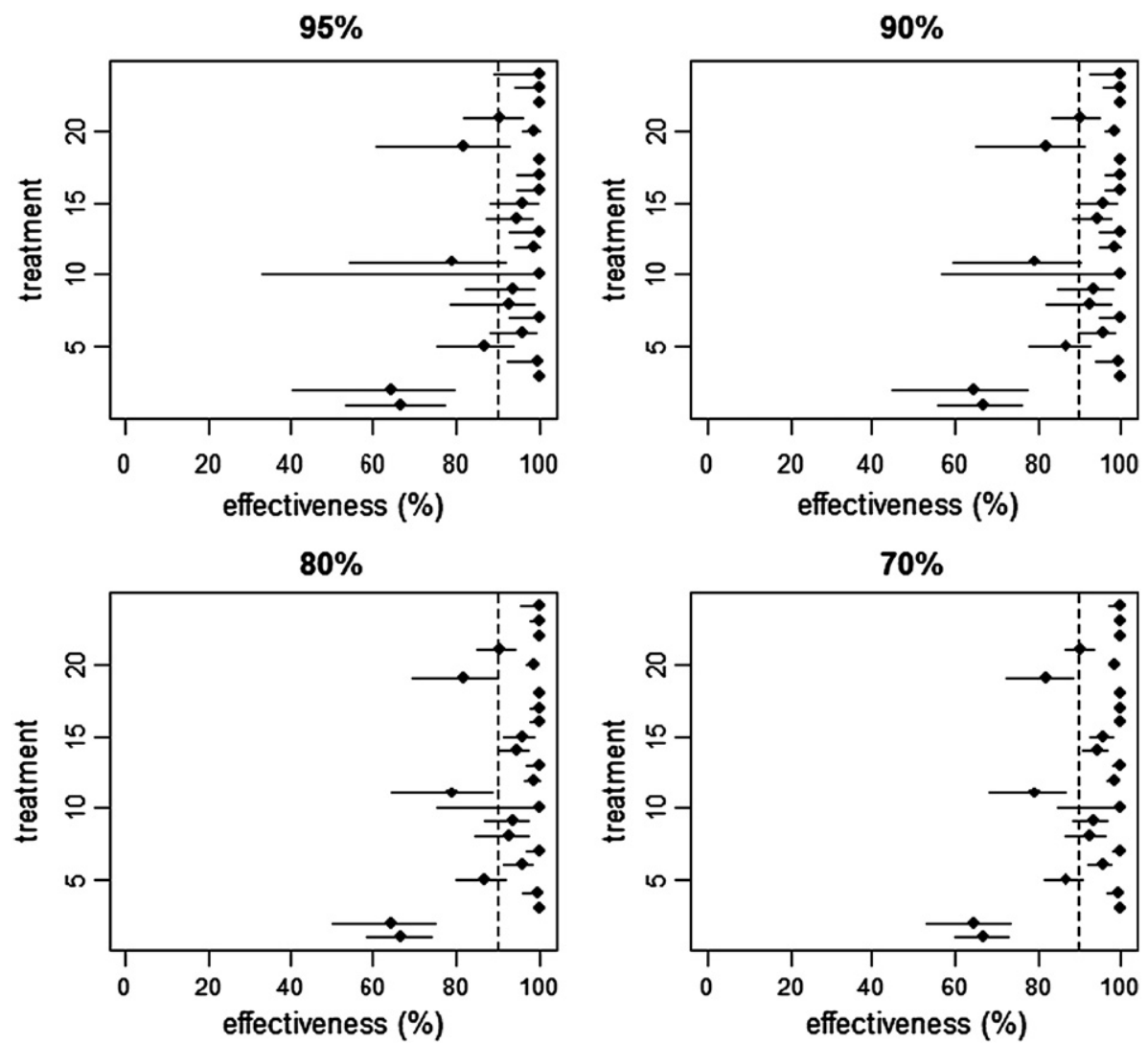

Fig. 4. Estimates of $L$. salmonis treatment effectiveness calculated with confidence levels of $70 \%, 80 \%, 90 \%$ and $95 \%$ in all treated pens ( $n=24$ ) using the quasi-Poisson method.
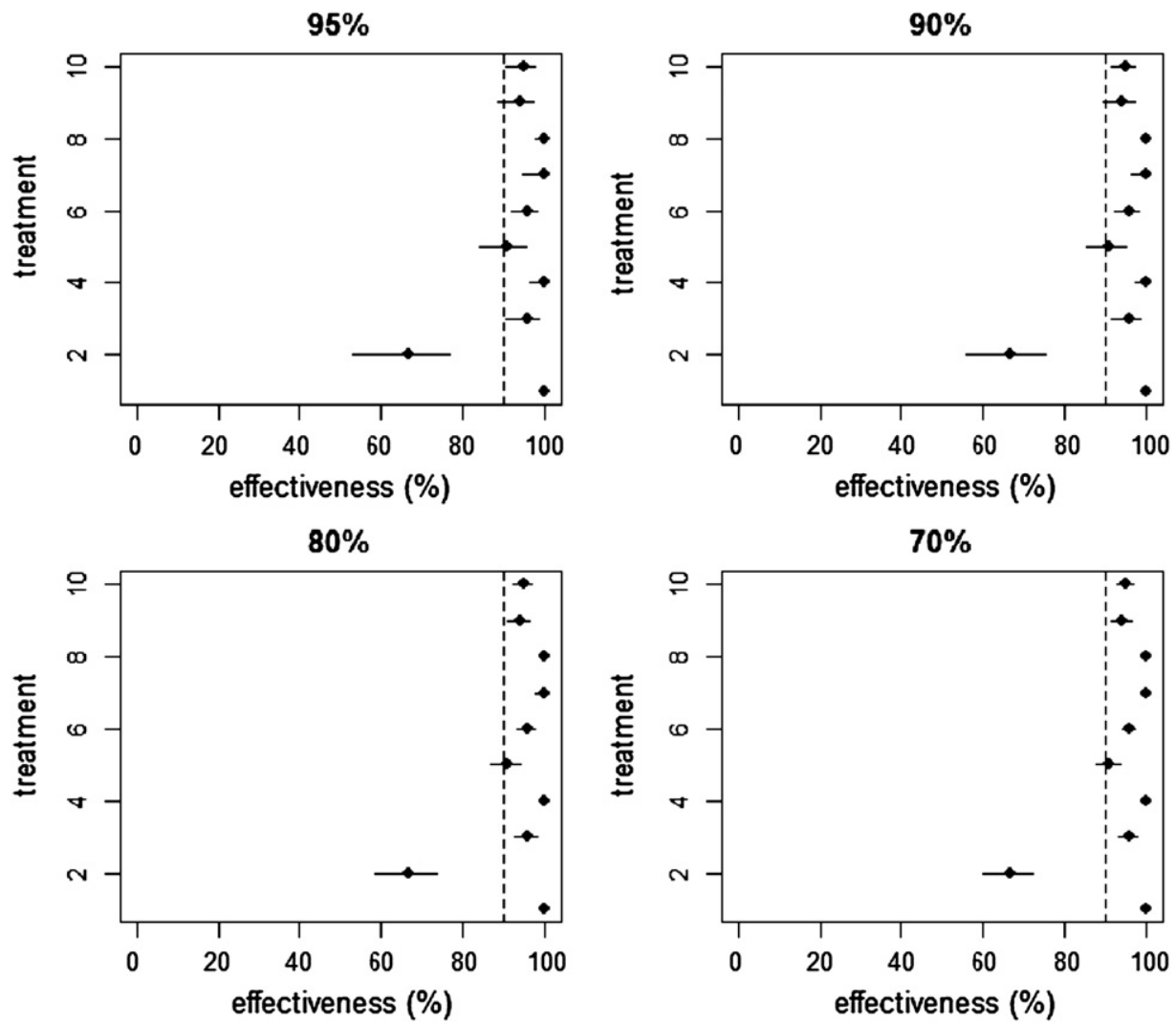

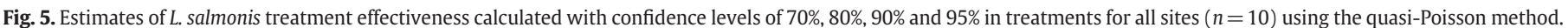


Table 4

Number of treatments against $L$. salmonis at pen level $(n=24)$ that were acceptable (A) and unacceptable (U) calculated with the "quasi-Poisson" method and using four different confidence levels.

\begin{tabular}{lllll}
\hline Result & $95 \%$ & $90 \%$ & $80 \%$ & $70 \%$ \\
\hline A & 11 & 12 & 14 & 15 \\
U & 13 & 12 & 10 & 9 \\
\hline
\end{tabular}

\section{Table 5}

Number of treatments against $L$. salmonis at farm level $(n=10)$ that were acceptable (A) and unacceptable (U) calculated with the "quasi-Poisson" method and using four different confidence levels.

\begin{tabular}{lllll}
\hline Result & $95 \%$ & $90 \%$ & $80 \%$ & $70 \%$ \\
\hline A & 7 & 7 & 8 & 8 \\
U & 3 & 3 & 2 & 2 \\
\hline
\end{tabular}

analysis of the factors related to treatment failure is beyond the scope of this study.

Finally, calculation of the treatment at site level was obtained by aggregating counts of mobiles from the available pens. The proportion of treatment failures was higher at pen than site level due to the average effect, and smaller variance from adding multiple pens and using a larger sample size. Sample size is a major determinant on the width of the confidence interval. Hence, appropriate sample sizes need to be calculated for estimations of effectiveness. Recommendations around sample size are challenging and beyond the objectives of this study, as additional theoretical (statistical power, for example) and practical issues (feasibility or cost associated with sampling, for example), that are not discussed in this study, need to be taken into consideration.

In this study, counts of mobiles were only available for a small proportion of pens on each site, and consequently, evaluation of treatment effect is likely not representative for the whole site as the sample of pens used was neither sufficient nor randomly selected. Hence, evaluation of the treatment at site level should include preferably all pens on the site to reduce the bias associated with the selection of a group of pens. This is achievable in Norway where large scale aquaculture uses fewer pens per site and monitoring of sea lice levels is performed in all pens at biweekly intervals.

In conclusion, it is more informative to provide measures of treatment effect together with confidence intervals. This will be useful for the early detection of changes in drug sensitivity and also for identifying technical problems associated with the application of baths. The proposed method can elucidate the current situation regarding the effectiveness of different delousing treatments in Norway and provide the basis for setting guidelines which are practical but also robust thus meeting the requirements of regulatory authorities and other stakeholders.

\section{Acknowledgments}

This research is part of a multi-disciplinary project (Topilouse) to improve the application of bath treatments against salmon lice. This research was funded by the Norwegian Research Council, the Fisheries and Aquaculture Research Fund, and our industry partners Marine Harvest, Salmar Farming, Pharmaq, Novartis, Rantex, Storvik Aqua and the Well Boat Owners Association. We wish to thank Dr. Anja B. Kristoffersen at the section of Epidemiology of the Norwegian Veterinary Institute, for its help and contribution with programming in $\mathrm{R}$, reading this manuscript and advice for improving before submission. We wish to thank Dr. Peder A. Jansen for constructive discussion and valuable comments that improved the manuscript.

\section{References}

Anderson, R.M., May, R.M., 1992. Infectious Diseases of Humans. Dynamics and Control Oxford University Press, Oxford, UK.

Baillie, M., Lees, F., Gettinby, G., Revie, C.W., 2009. The use of prevalence as a measure of sea lice burden: a case study of Lepeophtheirus salmonis on Scottish Atlantic salmon (Salmo salar L) farms. Journal of Fish Diseases 32, 15-25.

Beamish, R.J., Neville, C.M., Sweeting, R.M., Ambers, N., 2005. Sea lice on adult Pacific salmon in the coastal waters of Central British Columbia, Canada. Fisheries Research 76, 198-208.

Burka, J.F., Hammell, K.L., Horsberg, T.E., Johnson, G.R., Rainnie, D.J., Speare, D.J., 1997 Drugs in salmonid aquaculture-a review. Journal of Veterinary Pharmacology and Therapeutics 20, 333-349.

Burridge, L., Weis, J., Cabello, F., Pizarro, J., 2010. Chemical use in salmon aquaculture: a review of current practices and possible environmental effects. Aquaculture 306, 7-23.

Chaudhary, M.A., Stearns, S.C., 1996. Estimating confidence intervals for costeffectiveness ratios: an example form a randomized trial. Statistics in Medicine $15,1447-1458$.

Chen, Y.-H., Zhou, X.-H., 2006. Interval estimates for the ratio and difference of two lognormal means. Statistics in Medicine 25, 4099-4113.

Cimermanová, K., 2007. Estimation of confidence intervals for the log-normal means and for the ratio and the difference of log-normal means with application of breath analysis. Measurement Science Reviews 7, 31-36.

Coles, G.C., Bauer, C., Borgsteede, F.H.M., Geerts, S., Klei, T.R., Taylor, M.A., Waller, P.J., 1992. World Association for the Advancement of Veterinary Parasitology (W.A.A.V.P.) methods for the detection of antihelminthic resistance in nematodes of veterinary importance. Veterinary Parasitology 44, 35-44.

Committee for Veterinary Medicinal Products (CVMP), 2001). Guidelines on Statistical Principals for Veterinary Clinical Trials. EMEA/CVMP/816/00-FINAL. Available: http://www.ema.europa.eu, accessed in December 2011.

Committee for Veterinary Medicinal Products (CVMP, 2004). Guidelines on Specific Efficacy Requirements For Ectoparasiticides In Cattle. EMEA/CVMP/625/03/FINAL Available: http://www.ema.europa.eu, accessed in December 2011.

Corner, R.A., Davies, P.A., Cuthbertson, A.J.S., Telfer, T.C., 2011. A flume study to evaluate the processes governing retention of sea lice therapeutants using skirts in the treatment of sea lice infestation. Aquaculture 319, 459-465.

Denholm, I., Devine, G.J., Horsberg, T.E., Sevatdal, S., Fallang, A., Nolan, D.V., Powell, R. 2002. Analysis and management of resistance to chemotherapeutants in salmon lice, Lepeophtheirus salmonis (Copepoda: Caligidae). Pest Management Science 58, 528-536.

Directorate of Fisheries, 2009. Regulation for the Control of Sea Lice in Aquaculture Facilities. 2009-08-28 nr 1095. . Available: http://www.lovdata.no/for/sf/fi/xi20090818-1095.html, accessed in November 2011. (In Norwegian).

Fallang, A., Ramsay, J.M., Sevatdal, S., Burka, J.F., Jewess, P., Hammell, K.L., Horsberg, T.E. 2004. Evidence for occurrence of an organophosphate-resistant type of acetylcholinesterase in strains of sea lice (Lepeophtheirus salmonis Krøyer). Pest Management Science 60, 1163-1170.

Food, Drug Administration (FDA), 2010. Guidance for Industry. Non-inferiority Clinical Trials. Available:http://www.fda.gov, accessed in December 2011.

Fridell, F., 2009. Delousing salmon in large nets. Tarpaulins, skirts provide treatment confinement, distribution: Global Aquaculture Advocate November/December, pp. 41-43.

Friendly, M., 2000. Visualizing Categorical Data. SAS Institute, Cary, NC

Harrell, F.E., Davis, C.E., 1982. A new distribution-free quantile estimator. Biometrika 69, 635-640.

Hart, J.L., Thacker, J.R., Braidwood, J.C., Fraser, N.R., Matthews, J.E., 1997. Novel cypermethrin formulation for the control of sea lice on salmon (Salmo salar). The Veterinary Record 140, 179-181.

Hasler, M., Hothorn, L.A., 2008. Multiple Contrast Tests in the Presence of Heteroscedastic Data.

Hasler, M., Vonk, R., Hothorn, L.A., 2008. Assessing non-inferiority of a new treatment in a three-arm trial in the presence of heteroscedasticity. Statistics in Medicine 27 490-503.

Heuch, P.A., Revie, C.W., Gettinby, G., 2003. A comparison of epidemiological patterns of salmon lice, Lepeophtheirus salmonis, infections on farmed Atlantic Salmon, Salmo salar L., in Norway and Scotland. Journal of Fish Diseases 26, 539-551.

Heuch, P.A., Gettinby, G., Revie, C.W., 2011. Counting sea lice on Atlantic salmon farms empirical and theoretical observations. Aquaculture 2011, 3-4.

Hilbe, J.M., 2011. Negative Binomial Regression, Second ed. Cambridge University Press, Cambridge, UK.

Jones, M.W., Sommerville, C., Wootten, R., 1992. Reduced sensitivity of the salmon louse, Lepeophtheirus salmonis, to the organophosphate dichlorvos. Journal of Fish Diseases 15, 197-202.

Jones, P.G., Hammell, K.L., Dohoo, I.R., Revie, C.W., in review. Effectiveness of emamectin benzoate against sea lice (Lepeoptheirus salmonis) on farmed Atlantic salmon (Salmo salar) in the bay of Fundy, Canada from 2004-2008.

Lees, F., Baillie, M., Gettinby, G., Revie, C.W., 2008. The efficacy of emamectin benzoate against infestations of Lepeophtheirus salmonis on farmed Atlantic salmon (Salmo salar L) in Scotland, 2002-2006. PLoS One 3, e1549.

Marchiondo, A.A., Holdsworth, P.A., Green, P., Blackburn, B.L., Jacobs, D.E., 2007. World Association for the Advancement of Veterinary Parasitology (W.A.A.V.P.) guidelines for evaluating the efficacy of parasiticides for the treatment, prevention and control of flea and tick infestation on dogs and cats. Veterinary Parasitology 145, 332-344.

Norwegian Food Safety Authority (NFSA), 2010a. Summary of the Status of Salmon Lice in 2010. Available: http://www.mattilsynet.no/mattilsynet/multimedia/archive/ 
00066/Oppsummering_lakselu_66111a.pdf, accessed in December 2011. (in Norwegian).

Norwegian Food Safety Authority (NFSA), 2010b. Sea lice Regulations. Regulatory Guidelines for Aquaculture. Available:www.mattilsynet.no/smittevern_og bekjempelse/fisk/lakselus/rapportering/lakselussituasjonen_som_i_fjor_88883, accessed in September 2011. (in Norwegian).

Norwegian Food Safety Authority (NFSA), 2011. Regulations for Additional Requirements for the Treatment against Sea Lice as Determined for the First Half of 2012. Paragraph §3. Available: http://www.mattilsynet.no/mattilsynet/multimedia/ archive/00076/Forskrift_om_ytterli_76199a.pdf, accessed in January 2012. (in Norwegian).

R Development Core Team, 2008. R: A Language and Environment for Statistical Computing. R Foundation for Statistical Computing, Vienna, Austria.

Revie, C.W., Hollinger, H., Gettinby, G., Lees, F., Heuch, P.A., 2007. Clustering of parasites within cages of Scottish and Norwegian salmon farms: alternative sampling strategies using simulation. Preventive Veterinary Medicine 81, 135-147.

Schall, R., Luus, H.G., 2011. Comparative evaluation of ectoparasiticide efficacy: noninferiority and superiority assessment. Veterinary Parasitology 178, 173-179.

Schram, T.A., 1993. Supplementary descriptions of the developmental stages of Lepeophtheirus salmonis (Krøyer, 1837) (Copepoda: Caligidae). In: Boxshall, G.A., Defaye, D. (Eds.), Pathogens of Wild and Farmed Fish. Ellis Horwood, New York.

SEARCH Consortium, 2006. Sea Lice Resistance to Chemotherapeutants: A Handbook In Resistance Management. Available: (http://www.iacr.bbsrc.ac.uk/pie/searcj-EU/.2, accessed in November 2011.
Senn, S., 2007. Statistical Issues in Drug Development, Second ed. Wiley, University of Glasgow, UK.

Sevatdal, S., Horsberg, T.E., 2003. Determination of reduced sensitivity in sea lice (Lepeophtheirus salmonis Krøyer) against the pyrethroid deltamethrin using bioassays and probit modelling. Aquaculture 218, 21-31.

Smithson, M., 2003. Confidence Intervals. Sage Publications, Thousand Oaks, CA.

Steiger, J.H., Fouladi, R.T., 1997. Noncentrality interval estimation and the evaluation of statistical models. In: Harlow, L.L., Mulaik, S.A., Steiger, J.H. (Eds.), What if There Were No Significance Tests. Erlbaum, Mahwah, NJ, pp. 221-257.

Treasurer, J.W., Wadsworth, S., Grant, A., 2000. Resistance of sea lice, Lepeophtheirus salmonis (Kroyer), to hydrogen peroxide on farmed Atlantic salmon, Salmo salar L. Aquaculture Research 31, 855-860.

Tukey, J., 1977. Exploratory Data Analysis. Addison-Wesley, Reading, MA.

Tully, O., McFadden, Y., 2000. Variation in sensitivity of sea lice [Lepeophtheirus salmonis (Krøyer)] to dichlorvos on Irish salmon farms in 1991-92. Aquaculture Research 31, 849-854.

Venables, W.N., Ripley, B.D., 2002. Modern Applied Statistics. S. Springer, New York.

Ver Hoef, J.M., Boveng, P.L., 2007. Quasi-Poisson vs. negative binomial regression: how should we model overdispersed count data? Ecology 88, 2766-2772.

Vose, D., 2008. Risk Analysis: A Quantitative Guide. John Wiley \& Sons, Ltd., Chichester, UK.

Yaniv, I., Foster, D.P., 1997. Precision and accuracy of judgmental estimation. Journal of Behavior and Decision Making 10, 21-32.

Zeileis, A., Kleiber, C., Jackman, S., 2008. Regression models for count data in R. Journal of Statistical Software 27, 1-25. 